\title{
Hours Minutes Seconds Imputed
}

National Cancer Institute

\section{Source}

National Cancer Institute. Hours Minutes Seconds Imputed. NCI Thesaurus. Code C81213.

An imputed time that includes the hour, minute and second of observation. 